\title{
Impact of Next-to-Leading Order Contributions to Cosmic Microwave Background Lensing
}

\author{
Giovanni Marozzi, ${ }^{1}$ Giuseppe Fanizza, ${ }^{2}$ Enea Di Dio, ${ }^{3,4,5}$ and Ruth Durrer ${ }^{6}$ \\ ${ }^{1}$ Centro Brasileiro de Pesquisas Físicas, Rua Dr. Xavier Sigaud 150, Urca, CEP 22290-180 Rio de Janeiro, Brazil \\ ${ }^{2}$ Center for Theoretical Astrophysics and Cosmology, Institute for Computational Science, \\ University of Zürich, CH-8057 Zürich, Switzerland \\ ${ }^{3}$ INAF-Osservatorio Astronomico di Trieste, Via G. B. Tiepolo 11, I-34143 Trieste, Italy \\ ${ }^{4}$ SISSA-International School for Advanced Studies, Via Bonomea 265, 34136 Trieste, Italy \\ ${ }^{5}$ INFN_National Institute for Nuclear Physics, via Valerio 2, I-34127 Trieste, Italy \\ ${ }^{6}$ Université de Genève, Département de Physique Théorique and CAP, \\ 24 quai Ernest-Ansermet, CH-1211 Genève 4, Switzerland \\ (Received 28 December 2016; published 23 May 2017)
}

\begin{abstract}
In this Letter we study the impact on cosmological parameter estimation, from present and future surveys, due to lensing corrections on cosmic microwave background temperature and polarization anisotropies beyond leading order. In particular, we show how post-Born corrections, large-scale structure effects, and the correction due to the change in the polarization direction between the emission at the source and the detection at the observer are non-negligible in the determination of the polarization spectra. They have to be taken into account for an accurate estimation of cosmological parameters sensitive to or even based on these spectra. We study in detail the impact of higher order lensing on the determination of the tensor-to-scalar ratio $r$ and on the estimation of the effective number of relativistic species $N_{\text {eff }}$. We find that neglecting higher order lensing terms can lead to misinterpreting these corrections as a primordial tensorto-scalar ratio of about $O\left(10^{-3}\right)$. Furthermore, it leads to a shift of the parameter $N_{\text {eff }}$ by nearly $2 \sigma$ considering the level of accuracy aimed by future S4 surveys.
\end{abstract}

DOI: 10.1103/PhysRevLett.118.211301

Introduction.-In this Letter we discuss the effects of lensing of the temperature and polarization anisotropies of the cosmic microwave background radiation (CMB) beyond leading order. It is well known that gravitational lensing affects not only the CMB temperature fluctuations but also the polarization [1-4]. It actually transforms $E$ polarization (gradient type polarization) into $B$ polarization (curl-type polarization), which has to be subtracted from the primordial $B$ mode before it can be used to constrain the tensor-to-scalar ratio. This lensing-induced $B$ mode has already been measured [5-7]. The CMB, the most precise cosmological data set, therefore not only allows us to determine with high precision the fluctuations of the spacetime geometry and the matter distribution at the time of last scattering, but via lensing, it also allows us to determine the (integrated) fluctuations in the geometry at lower redshifts, mainly due to the growth of matter density perturbations. One of the important goals of future CMB experiments is to measure lensing out to the $\mathrm{CMB}$ with high precision and generate precise lensing maps $[5,8-13]$.

The effects of lensing in the CMB are quite substantial. For $\ell \sim 800$ its effect on temperature anisotropies is about $1 \%$ and it rises to $10 \%$ by $\ell \sim 2200$. For $\ell>5000$ it dominates over the primordial signal. Present analysis is mainly based on the first order perturbations of the CMB from lensing. The Taylor series in the first order deflection angle, however, has to be resummed. This can be performed exactly assuming a Gaussian deflection angle [3,4]. It turns out that this resummation is quite important, e.g., for cosmological parameter estimation with CAMB [14] or CLASS $[15,16]$, and already for present experiments like Planck [17]. It is therefore reasonable to ask for a systematic next-order calculation including especially also non-Gaussian contributions (e.g., from an odd number of deflection angles), which are present at higher order. This has been attempted in Ref. [18] for the temperature and in Refs. [19-21] for both the temperature and the polarization. In particular, in Ref. [18] the contribution from the nonGaussian post-Born terms was evaluated for the first time and its dominance over other post-Born corrections has been shown. Nevertheless, all the new contributions beyond the leading order lensing of the CMB temperature anisotropies, as found in these references, are quite small.

In this Letter, starting from the results of Ref. [22], we study the effect of higher order lensing of the CMB temperature and polarization on the evaluation of cosmological parameters. In Ref. [22] the beyond leading order lensing of the CMB polarization was evaluated in full generality, going beyond the results given in Refs. [19-21]. Especially, a new effect has been considered in Ref. [22], which was overlooked previously, and which actually dominates the next-to-leading order correction for $B$ 
polarization. The physical origin of this new effect is quite simple: the CMB polarization tensor is parallel transported along a perturbed lightlike geodesic. Therefore, we see the photons in a direction that differs from the one into which they have been emitted. But parallel transport of a tensor also rotates its principal axes. It is well known that for purely scalar lensing this rotation vanishes, but if the lens map has a curl component [23], this leads to a rotation of the polarization direction. It also induces a small additional deflection, but it has been shown in Ref. [21] that this is very subdominant. Here, we show how the effect coming from the rotation of the polarization axis, together with the other higher order contributions, can have a substantial impact on the determination of cosmological parameters.

The new effect described above is especially important since the curl lensing transforms $E$ polarization into $B$ polarization and, together with the other higher order lensing effects, modifies the $B$ modes from lensing by up to about $0.1 \%$ for $\ell<1500$ and enhances them by nearly $2 \%$ for $\ell \simeq 3500$ (see Ref. [22]). These contributions are relevant when performing the lensing subtraction of $B$ modes in order to recover the primordial signal from inflationary gravitational waves. If discovered, this signal, and especially the tensor-to-scalar ratio $r$, will provide the energy scale of inflation and allow us a glimpse of the physics at the highest energies ever observed, many orders of magnitude higher than the energy achieved at the LHC at CERN. It is therefore very important to identify all possible foregrounds that might hamper the detection of $r$.

But even independent of primordial $B$ modes, the detection of the lensing curl mode in $B$ polarization will be a formidable test of general relativity (GR) as it is a measurement of gravitational "frame dragging," an effect detected for the first time with Gravity Probe B [24] from the gravitational field of the rotating Earth. Seeing the lensing-curl rotation of $B$ mode polarization tests frame dragging on cosmological scales. This shows once more, that cosmology offers many opportunities to actually test GR on large scales.

Higher order lensing corrections of the polarization spectra are not just important for the determination of the tensor-to-scalar ratio. As we show in this Letter, they are also relevant for cosmological parameter estimation, especially at the level of precision aimed in a future survey [12].

Methods.-Here, we present the basic ideas and sketch the derivation of the results obtained in Ref. [22]. A much more detailed description is provided in Ref. [22], where the analytical expressions are presented and a comparison of the magnitude of the effects with cosmic variance is performed.

When observing a scalar quantity in the sky (e.g., the temperature anisotropy) in a direction $\boldsymbol{\theta}$, we have to take into account that this direction is deflected by lensing and the true direction of the source is $\boldsymbol{\theta}+\delta \boldsymbol{\theta}$. The observed temperature anisotropy, let us call it $\tilde{\mathcal{M}}$, is therefore related to the anisotropy on the last scattering surface, which we denote $\mathcal{M}$, by

$$
\tilde{\mathcal{M}}(\boldsymbol{\theta})=\mathcal{M}(\boldsymbol{\theta}+\delta \boldsymbol{\theta}) .
$$

The deflection is caused by foreground structures and can be considered as uncorrelated with the temperature anisotropy. Since $\langle\delta \boldsymbol{\theta}\rangle=0$, a systematic expansion yielding the leading and next-to-leading corrections to the power spectrum has to go up to third order in the deflection angle and must include terms with up to four angles,

$$
\begin{aligned}
\tilde{\mathcal{M}}(\boldsymbol{\theta})= & \mathcal{M}(\boldsymbol{\theta})+\sum_{i=1}^{3} \delta \theta^{a(i)} \nabla_{a} \mathcal{M} \\
& +\frac{1}{2} \sum_{i+j \leq 4} \delta \theta^{a(i)} \delta \theta^{b(i)} \nabla_{a} \nabla_{b} \mathcal{M} \\
& +\frac{1}{6} \sum_{i+j+k \leq 4} \delta \theta^{a(i)} \delta \theta^{b(j)} \delta \theta^{c(k)} \nabla_{a} \nabla_{b} \nabla_{c} \mathcal{M} \\
& +\frac{1}{24} \delta \theta^{a(1)} \delta \theta^{b(1)} \delta \theta^{c(1)} \delta \theta^{d(1)} \nabla_{a} \nabla_{b} \nabla_{c} \nabla_{d} \mathcal{M}
\end{aligned}
$$

The superscripts in parentheses indicate the order at which the deflection angle has to be taken, the Latin indices $a$ to $d$ go over the two directions of the sphere, and the $\nabla_{a}$ are covariant derivatives on the sphere. Introducing the Weyl potential as the mean of the Bardeen potentials [4]

$$
\Phi_{W}=\frac{1}{2}(\Phi+\Psi),
$$

the deflection angle can be determined recursively as the weighted integral of $\Phi_{W}$; it is given in Ref. [25].

To obtain the spectra one then converts Eq. (2) into spherical harmonic space. Details are presented in Ref. [18].

At second order the Jacobi map, which describes the evolution of the geodesic deviation vector, not only contains expansion and shear but also a rotation due to the induced vector mode. This represents the rotation $\beta$ of the geodesically transported Sachs basis of the screen. The CMB polarization tensor given in terms of the Stokes parameters $Q$ and $U$ as $\mathcal{P}_{ \pm}=Q \pm i U$, which has helicity \pm 2 , transforms with $e^{ \pm 2 i \beta}$ under this rotation. It is well known that a rotation of the polarization pattern transforms $E$ modes into $B$ modes and vice versa $[4,26]$. To take this effect into account up to next-to-leading order, we need in principle the rotation angle up to fourth order. But $\beta^{(0)}=0$ and for scalar perturbations also $\beta^{(1)}=0$. The only terms from rotation in the power spectrum are therefore of the form $\left[\beta^{(2)}\right]^{2}$ and we can neglect $\beta^{(3)}$ and $\beta^{(4)}$. The expectation value of terms containing only one rotation angle have to vanish for parity reasons. In Ref. [22] we obtain 


$$
\begin{aligned}
\beta^{(2)}(\boldsymbol{\theta})= & 2 \epsilon^{a b} \int_{0}^{r_{s}} d r \frac{r_{s}-r}{r_{s} r} \nabla_{a} \nabla^{c} \Phi_{W}(r) \\
& \times \int_{0}^{r} d r_{1} \frac{r-r_{1}}{r r_{1}} \nabla_{b} \nabla_{c} \Phi_{W}\left(r_{1}\right),
\end{aligned}
$$

where $\Phi_{W}(r) \equiv \Phi_{W}\left(\eta_{0}-r, r \boldsymbol{\theta}\right)$. This rotation angle is identical to the rotation angle in the lens map usually called $\omega$, even if these angles have slightly different physical meanings, as shown in Ref. [23] for first order vector and tensor perturbations. Indeed, $\omega$ refers to a bundle of light rays and describes the rotation of an image along the line of sight, whereas $\beta$ acts also on a single photon yielding the rotation of a geodesically transported vector. Nevertheless, in Ref. [22] a direct evaluation of $\beta$ by computing the Sachs basis, in geodesic light-cone gauge (see Refs. [27-29]), and the straightforward derivation of $\omega$ as the antisymmetric part of the amplification matrix, shows the equality of the two angles.

At next-to-leading order, we have three contributions: pure post-Born terms due to higher order deflection angles, the large-scale structure (LSS) contribution to higher order deflection angles (obtained by expanding the Weyl potential beyond linear order), and, for the polarization spectra, corrections from the rotation angle $\beta$. [The contribution from the rotation can be further divided into two parts, one of them is a "constant" contribution that just leads to an overall shift of $\Delta C_{\ell}^{X X} / C_{\ell}^{X X}$ (see Ref. [22] for details). This part is negligible in cosmological parameter estimation and we do not consider it in this Letter.] The general expression for a lensed spectrum can be written as

$$
\tilde{C}_{\ell}^{X Y}=\tilde{C}_{\ell}^{X Y(1)}+\Delta C_{\ell, p B}^{X Y}+\Delta C_{\ell, \mathrm{LSS}}^{X Y}+\Delta C_{\ell, \beta}^{X Y},
$$

where $\tilde{C}_{\ell}^{X Y(1)}$ denotes the unlensed $C_{\ell}^{X Y}$ plus the well known resummed correction from the first order deflection angle $[1,3,4]$. The post-Born and LSS contributions can be divided into two groups. Following the formalism of Refs. [18,22], we have a "second group" that involves two point correlation functions of deflection angles (first $\times$ third or second $\times$ second order), and a "third group" that contains three point correlation functions of deflection angles (first $\times$ first $\times$ second order). Hereafter, we use the Halofit model (see Refs. [30,31]) for the matter power spectrum. The second group of LSS corrections is then included in the leading terms. Therefore, this contribution is not added separately. Beyond the Halofit model, our results can be generalized also to fitting formulae for the bispectrum [32]. These fitting formulae can then be used to evaluate the full higher-order effects on the bispectrum [33,34].

Results.-In Ref. [22] we have evaluated the corrections summarized in Eq. (5) to the temperature and the polarization spectra. In particular, the new contribution coming from the rotation turns out to be non-negligible for the

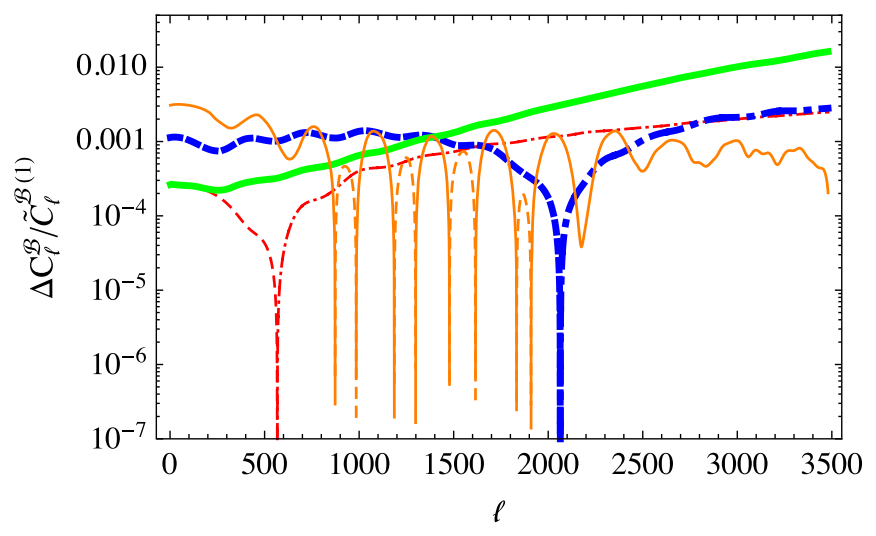

FIG. 1. Next-to-leading order corrections to $B$ modes. We show the correction from the post-Born second group (thin dot-dashed red), the post-Born third group (thick dot-dashed blue), and the LSS (thin orange), and the contribution coming from the rotation angle $\beta^{(2)}$ (thick green); negative values are dashed. All functions are normalized to the resummed first order deflection angle $\tilde{C}_{\ell}^{\mathcal{B}(1)}$, which is included in standard CMB codes.

$E$-mode spectrum, and leading at high $\ell$ for the $B$-mode spectrum, see Ref. [22] for details. In Fig. 1 we show the most interesting case, i.e., the ratio of the new terms to the standard (first order resummed) lensing-induced $B$ mode. As the figure clearly shows, the contribution from rotation not only cannot be neglected but actually dominates at $\ell>1500$. Different higher order contributions dominate in different $\ell$ ranges. At low $\ell$ the non-Gaussian post-Born contribution and the LSS contribution dominate with different signs and they partially cancel. This is particularly relevant for $\ell<300$, the range used to infer the value of $r$, see, e.g., Ref. [6]. The importance of including higher order lensing when performing the lensing subtraction of $B$ modes, in order to recover the primordial signal from inflationary gravitational waves, is evident from Fig. 2. There we compare the $B$ mode from lensing with a possible primordial signal for different values of $r$ and including the leading correction and the total higher order contribution (sum of the post-Born, LSS, and rotation contributions). Clearly, the higher order lensing correction can be misinterpreted for a primordial signal at the level of $r \simeq 10^{-3}$ or more from $\ell>200$, even if the leading terms are correctly subtracted. Namely, if we neglect this correction we could erroneously measure a primordial signal using future surveys, which plan to have a level of accuracy that would be sensitive to $r \simeq O\left(10^{-3}\right)$ [12]. The impact of higher order lensing on the $r$ measurement is lower for $\ell$ up to $\ell_{\max }=200$, but it is still at the level of $r \simeq 4.5 \times 10^{-4}$ for $\ell=150$ and to measure $r \simeq 10^{-3}$ at $3 \sigma$ the higher order lensing correction has to be subtracted.

From Fig. 1 we infer that at small $\ell$ the correction to the lensing contamination can be at the level of $0.1 \%$. Meanwhile, at high $\ell \gtrsim 2500$ it adds a contribution at the percent level to the lensing power spectrum. It is important 


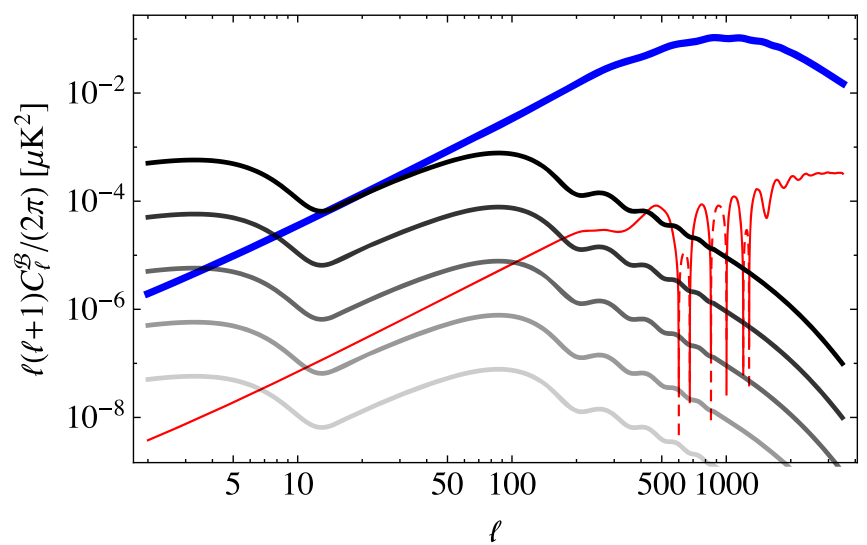

FIG. 2. The first order resummed lensing correction to the $B$ modes of the CMB (thick blue line) and the additional correction from the full higher order contribution (post-Born + LSS + rotation contributions, thin red line) are compared. The gray lines refer to primordial $B$ modes with no lensing for different values of $r, r=10^{-2}, 10^{-3}, 10^{-4}, 10^{-5}, 10^{-6}$ from top to bottom. Negative values are dashed.

to remember that here we show the power spectra; in the amplitude of the fluctuations this contribution is more than $10 \%$.

Let us also consider another example to show the importance of the higher order lensing correction in the determination of cosmological parameters with the future CMB survey. We discuss the effect of higher order lensing for the determination of the effective number of relativistic species $N_{\text {eff }}$. In Fig. 3 we show the theoretical bias introduced in cosmological parameter estimation if higher order $\mathrm{CMB}$ lensing contributions are neglected. Considering as parameters the effective number of relativistic species and the dark matter density parameter $\omega_{\mathrm{cdm}}=h^{2} \Omega_{\mathrm{cdm}}$, and keeping all the other parameters fixed

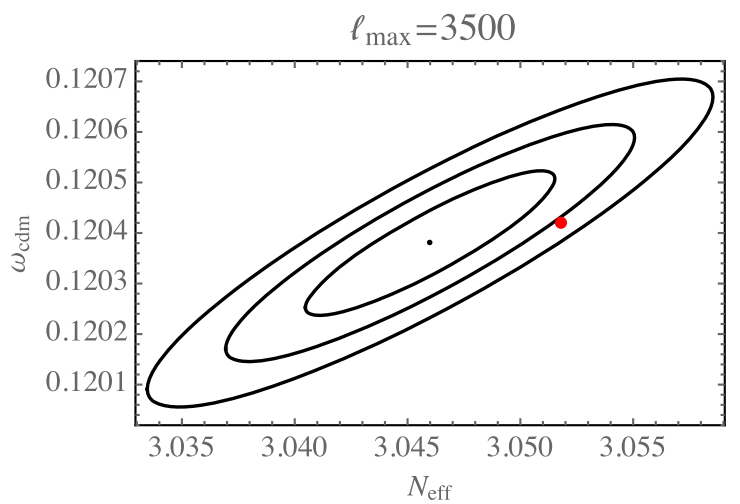

FIG. 3. The bias introduced in cosmological parameter estimation if subleading $\mathrm{CMB}$ lensing effects are neglected. We consider multipoles up to $\ell_{\max }=3500$ for $T T, E E$, and $T E$ and $\ell_{\max }=1500$ for $B B$ spectra, for an ideal cosmic variance limited survey with sky coverage $f_{\text {sky }}=0.75$ for $T T, E E$, and $T E$ and $f_{\text {sky }}=0.5$ for $B B$ spectra, keeping all the parameters not shown fixed at their fiducial values. at their fiducial values, we have performed a Fisher matrix analysis considering temperature, $E$ modes, and $T E$ correlation power spectra up to $\ell=3500$ for a cosmic variance limited survey with sky coverage $f_{\text {sky }}=0.75$ for $T T, E E$, and $T E$ and $f_{\text {sky }}=0.5$ for $B B$ spectra. Neglecting higher order lensing contributions leads to a non-negligible shift (about $2 \sigma$ ) of the measured parameters with respect to their true value. The shift becomes even larger for the ideal case of full sky coverage, i.e., well beyond $2 \sigma$. In performing this analysis we have in mind an S4 CMB experiment with a conservative value of the attainable upper bound of $C_{\ell} \approx 2.5 \times 10^{-7} \mu \mathrm{K}^{2}$ [12]. In this case we can reach $\ell \simeq$ 3500 in the $E$-mode spectrum (and even higher values for the temperature and $T E$ power spectra). We also consider $B$ modes up to $\ell \simeq 1500$; however, they carry less information. Furthermore, considering that using a Fisher matrix technique approximates the spectra as Gaussian fields, which underestimates the contribution to the covariance induced by the $B$ mode (see, e.g., Ref. [35]), the true constraining power of the $B$ mode is probably even smaller. Keeping the other cosmological parameters fixed at their fiducial values means that we assume that they are determined with good accuracy by other cosmological probes. When marginalizing also over $H_{0}$ the shift reduces to somewhat more than $1 \sigma$, while marginalizing over all cosmological parameters reduces the shift below $1 \sigma$.

These findings demonstrate the importance of higher order lensing for a very accurate determination of parameters like the effective number of relativistic species. Moreover, if we consider the optimistic upper bound of $C_{\ell} \approx 10^{-8} \mu \mathrm{K}^{2}$ achievable by S4 CMB experiments, we can go to larger values of $\ell$ and the shift becomes even more significant. In this optimistic case the power spectra are measured roughly at the cosmic variance accuracy up to the values of $\ell_{\max }$ considered in the Fisher matrix analysis.

Clearly, the Fisher matrix is an approximation and a full Monte Carlo analysis would be more reliable. The Fisher matrix can only be trusted when the parameter shift is small (when it is large it can differ significantly from a Monte Carlo result [36]). However, the present analysis is a proof of principle demonstrating that cosmological parameters inferred from S4-precision CMB data can shift by more than $1 \sigma$ due to higher order lensing terms.

Conclusions.-In this Letter we have shown that next-toleading order lensing corrections to CMB temperature and polarization spectra can be crucial in cosmological parameter estimation from $\mathrm{CMB}$ data, and cannot be neglected in future $\mathrm{CMB}$ experiments.

In particular, lensing at higher order significantly modifies $B$-mode polarization. Even though the signal is quite small, it has to be included for lensing reconstruction of primordial $B$ modes. If not, one erroneously measures a primordial tensor-to-scalar ratio at the level of $10^{-3}$ in the multipole range $200<\ell<300$ (although at larger scales, lower $\ell$, the impact is smaller). 
On smaller scales, $\ell>1500$ the new lensing correction is rather large and it is dominated by the rotation induced by the curl part of the lensing potential. This signal is purely second order in perturbation theory and therefore nonGaussian, which can help to identify it. Measuring this spin-1 signal will be possible with S4-precision experiments (see Ref. [37]) and it represents a measure of frame dragging in the Universe. Finding the best way to measure $\beta$ is an interesting open problem. The proposal of Ref. [26] does not work in this case since the random rotation angle $\beta$ is virtually uncorrelated with the CMB anisotropies and polarization and does not lead to nonvanishing $E B$ or $T B$ cross spectra.

To conclude, not only are the correction of $B$ modes important but, as we have shown in Fig. 3, the higher order lensing corrections to $T E$ and $E E$ have to be included to measure, for example, the effective number of relativistic species at the level of accuracy aimed at by future surveys, see Ref. [12]. The higher order contribution to the $E$ spectrum is partly degenerate with the modifications from a change in the effective number of relativistic species. Neglecting the next-to-leading order corrections to $E$ polarization leads to a shift of the cosmological parameter $N_{\text {eff }}$ of nearly $2 \sigma$ when considering multipoles up to $\ell=3500$, see Fig. 3 .

We thank Giulio Fabbian and Tom Crawford for helpful discussions. G. M. wishes to thank $\mathrm{CNPq}$ for financial support. G. F. is supported by a Consolidator Grant of the European Research Council (ERC-2015-CoG Grant No. 680886). E. D. is supported by the ERC Starting Grant cosmoIGM and by the Istituto Nazionale di Fisica Nucleare/Padova 51 INDARK grant. R. D. acknowledges support from the Swiss National Science Foundation.

[1] U. Seljak, Astrophys. J. 463, 1 (1996).

[2] C. M. Hirata and U. Seljak, Phys. Rev. D 68, 083002 (2003);

[3] A. Lewis and A. Challinor, Phys. Rep. 429, 1 (2006).

[4] R. Durrer, The Cosmic Microwave Background (Cambridge University Press, Cambridge, England, 2008).

[5] P. A. R. Ade et al. (POLARBEAR Collaboration), Phys. Rev. Lett. 113, 021301 (2014).

[6] P. A. R. Ade et al. (BICEP2, Planck Collaboration), Phys. Rev. Lett. 114, 101301 (2015).

[7] P. A. R. Ade et al. (Planck Collaboration), Astron. Astrophys. 596, A102 (2016).

[8] K. T. Story et al. (SPT Collaboration), Astrophys. J. 810, 50 (2015).

[9] P. A. R. Ade et al. (Planck Collaboration), Astron. Astrophys. 594, A15 (2016).
[10] P. A. R. Ade et al. (BICEP2, Keck Array Collaboration), Astrophys. J. 833, 228 (2016).

[11] B. D. Sherwin et al. (ACT Collaboration), arXiv:1611.09753.

[12] K. N. Abazajian et al. (CMB-S4 Collaboration), arXiv: 1610.02743.

[13] E. Di Valentino et al. (CORE Collaboration), arXiv: 1612.00021.

[14] A. Lewis, A. Challinor, and A. Lasenby, Astrophys. J. 538, 473 (2000).

[15] J. Lesgourgues, arXiv:1104.2932.

[16] D. Blas, J. Lesgourgues, and T. Tram, J. Cosmol. Astropart. Phys. 07 (2011) 034.

[17] P. Ade et al. (Planck Collaboration), Astron. Astrophys. 594, A13 (2016).

[18] G. Marozzi, G. Fanizza, E. Di Dio, and R. Durrer, J. Cosmol. Astropart. Phys. 09 (2016) 028.

[19] S. Hagstotz, B. M. Schäfer, and P. M. Merkel, Mon. Not. R. Astron. Soc. 454, 831 (2015).

[20] G. Pratten and A. Lewis, J. Cosmol. Astropart. Phys. 08 (2016) 047.

[21] A. Lewis and G. Pratten, J. Cosmol. Astropart. Phys. 12 (2016) 003.

[22] G. Marozzi, G. Fanizza, E. Di Dio, and R. Durrer, arXiv:1612.07263.

[23] L. Dai, Phys. Rev. Lett. 112, 041303 (2014).

[24] C. W. F. Everitt et al., Phys. Rev. Lett. 106, 221101 (2011)

[25] G. Fanizza, M. Gasperini, G. Marozzi, and G. Veneziano, J. Cosmol. Astropart. Phys. 08 (2015) 020.

[26] M. Kamionkowski, Phys. Rev. Lett. 102, 111302 (2009).

[27] M. Gasperini, G. Marozzi, F. Nugier, and G. Veneziano, J. Cosmol. Astropart. Phys. 07 (2011) 008.

[28] G. Fanizza, M. Gasperini, G. Marozzi, and G. Veneziano, J. Cosmol. Astropart. Phys. 11 (2013) 019.

[29] G. Fanizza and F. Nugier, J. Cosmol. Astropart. Phys. 02 (2015) 002.

[30] R. E. Smith, J. A. Peacock, A. Jenkins, S. D. M. White, C. S. Frenk, F. R. Pearce, P. A. Thomas, G. Efstathiou, and H. M. P. Couchmann (VIRGO Consortium), Mon. Not. R. Astron. Soc. 341, 1311 (2003).

[31] R. Takahashi, M. Sato, T. Nishimichi, A. Taruya, and M. Oguri, Astrophys. J. 761, 152 (2012).

[32] H. Gil-Marin, C. Wagner, F. Fragkoudi, R. Jimenez, and L. Verde, J. Cosmol. Astropart. Phys. 02 (2012) 047.

[33] T. Namikawa, Phys. Rev. D 93, 121301 (2016).

[34] V. Böhm, M. Schmittfull, and B. D. Sherwin, Phys. Rev. D 94, 043519 (2016).

[35] K. M. Smith, W. Hu, and M. Kaplinghat, Phys. Rev. D 74, 123002 (2006).

[36] W. Cardona, R. Durrer, M. Kunz, and F. Montanari, Phys. Rev. D 94, 043007 (2016).

[37] G. Fabbian, M. Calabrese, and C. Carbone, arXiv: 1702.03317. 